erkennen. Die Leitung der Geburt aus fetaler Sicht wird sich in Zukunft im wesentlichen nach diesen Blutbefunden richten. Wer sich mit den Vorteilen dieser Methode in der Praxis vertraut gemacht hat, mag nicht mehr auf sie verzichten.

Die Indikationen für die Mikroblutuntersuchungen am Feten sind nach den neuesten Erfahrungen ergänzt worden (S. 771). Den mittleren und kleineren Kliniken, die Mikroblutuntersuchungen am Feten mit möglichst geringem Aufwand betreiben wollen, ist die pH-Messung (S. 752, 770-773) zu empfehlen, die jeder Kreißsaalassistent ausführen kann und die nur etwa 30 Sekunden in Anspruch nimmt. Genaue Angaben über die dabei zu beachtenden Grenzwerte finden sich auf S. 773.

Für kritische Ratschläge und wertvolle Anregungen bin ich zu besonderem Dank verpflichtet Herrn Prof. H. Kräubig, Herrn Prof. H. Kremling, den Herren Priv. Doz. Dr. H. Hoffbauer, Dr. E. J. Hickl, Dr. Potel sowie Herrn Dr. H. Ludwig. Ebenso habe ich meiner Frau zu danken, die das Kapitel Morbus haemolyticus neonatorum bearbeitet, Korrekturen gelesen und das Register angefertigt hat. Herr cand. med. J. Dudenhausen hat beim Lesen der Korrekturen geholfen, wofür ich ihm meinen Dank ausspreche.

1 Berlin 19

W. Pschyrembel

Rüsternallee 45

\title{
Zur 12. und 13. Auflage
}

Die 11. Auflage war nach knapp einem Jahr vergriffen. Eine Neubearbeitung war in der verhältnismäßig kurzen Zeit nicht möglich und auch nicht notwendig. So erscheinen die 12. und 13. Auflage unverändert als Nachdruck der 11. Auflage.

1 Berlin 19

W. Pschyrembel

Rüsternallee 45 